\title{
STABILITY CHARACTERISTICS OF CYLINDRICAL FIBRES IN AN ELECTRODYNAMIC BALANCE DESIGNED FOR SINGLE PARTICLE INVESTIGATION
}

\author{
E. Hesse, Z. Ulanowski, and P. H. Kaye
}

Science and Technology Research Centre, University of Hertfordshire, Hatfield, Hertfordshire AL10 9AB, UK

\begin{abstract}
An electrodynamic balance (EDB) of the double ring double disc type has been designed for studying single microparticles. It allows electrostatic particle injection and recovery as well as alignment of elongated particles. The stability characteristics of cylindrical fibers and their alignment in this EDB are investigated. Angular as well as translational oscillations are observed, each associated with separate but analogous stability criteria.
\end{abstract}

Keywords: electrodynamic balance, fiber, alignment, stability, levitation 


\section{INTRODUCTION}

The electrodynamic balance (EDB) has been used in a wide range of single particle studies, e.g. mass and charge measurements, evaporation / condensation studies, light scattering measurements, and photoemission experiments on both liquid and solid particles (Davis, 1997).

For these purposes various designs of the actual trap as well as particle injection devices have been developed. In most of these applications large numbers of particles are available. For example, droplets are produced by vibrating orifice aerosol generators or by using a hypodermic needle. Solid sodium chloride or sodium sulfate particles were formed by allowing larger aqueous solution droplets to evaporate, thereby forming a solid residue (Aardahl et al., 1997). Graphite particles of ca. $3 \mu \mathrm{m}$ size were injected into an EDB by pushing a pin through a cone shaped reservoir (Arnold and Hessel, 1985). Sasse et al. (1996) trapped carbon and quartz spheres of 100-200 $\mu \mathrm{m}$ diameter by bringing them into contact with an electrostatically charged plastic rod which, when moved close to the middle rings of a four-ring EDB and vibrated, released some particles causing them to levitate in the EDB.

These techniques are less suitable for experiments in which only small numbers of particles are available or when individual, unique particles must be investigated. Likewise, when dealing with single particles it is often desirable to recover particles from the trap so that further examination, for example by SEM, can be carried out. Arnold and Folan (1986) collected $10 \mu \mathrm{m}$ glycerol droplets from an EDB by inserting a $1 \mathrm{~mm}$ quartz fiber into the trap and adjusting the dc voltage so that the particle collided with the fiber and stuck to it. Ejecting a particle upward through an aperture in the top plate of an EDB and onto a flat quartz disk was considered by Vedder (1963). In the study presented here, a method for single particle injection using a fine tungsten needle is developed. The same needle is also used to recover single solid particles after the levitation experiment.

Once the particle is trapped, it should be kept stable, by which we mean that after an initial period of damping due to the aerodynamic drag force it should cease to oscillate. The existence of stability boundaries, i.e. certain values of ac amplitude and frequency, specific to the balance geometry as well as mass, charge and size of the particle, at which the suspended particle starts to oscillate, was already described in the early works of Straubel (1956), and Wuerker et al. (1959). Wuerker et al. (1959) introduced the hyperboloidal trap and solved the equations of motion for the drag-free case, which are homogeneous Mathieu-equations. Frickel et al. (1978) inserted a drag term and solved the equations of motion numerically, as Ataman and Hanson (1960) did when modeling Straubel's balance consisting of a ring electrode and two flat endcap electrodes. Equations of motion were solved exclusively for translational motion, mostly for spherical particles. Recently, ellipsoidal particles were investigated (Zheng et al., 2000; Laucks et al., 2000). A general theory for translational motion stability of electrodynamic balances, which have a non-vanishing quadrupole field term, was derived by Hartung and Avedisian (1992). Generalized equations of motion were given, which allow the calculation of stability boundaries for particle motion in axial and radial direction for any EDB, if two constants characterizing the balance are known. In the case of elongated particles, which is investigated here, an additional stability boundary exists. This boundary marks the onset of angular oscillations. In the later sections of this paper the stability boundaries of cylindrical particles are investigated by solving the equations of motion and comparing them to experimental results obtained for glass fibers.

\section{THE SET-UP of the EDB}

A diagram of the electrodynamic balance is shown in Fig. 1. It is a modified double ringdouble disc EDB consisting of two truncated cones as ac electrodes and two disks made of thin glass with a semi-transparent conducting coating and metallic cylindrical extensions as dc electrodes. The dc electrodes have cylindrical extensions in order to reach a high levitation strength constant as well as to shield the chamber from external fields, especially from the ac supply lead. The size of the chamber is kept small in order to allow particle observation through high-resolution objectives with a working distance of $14 \mathrm{~mm}$. The endcaps of the dc electrodes have a distance of $11.5 \mathrm{~mm}$ from the balance center. The upper endcap has a central aperture $1.5 \mathrm{~mm}$ in diameter for particle injection. The levitation strength constant $c_{0}=33.58 \mathrm{~m}^{-1}$ was determined from numerical calculation of the field strength $E_{\mathrm{dc}}$ at the center of the balance at a certain dc voltage $V_{\mathrm{dc}}\left(c_{0}=E_{\mathrm{dc}} / V_{\mathrm{dc}}\right)$. For the alignment of nonspherical particles, the ac electrodes can be rotated around an axis perpendicular to the trap axis and intersecting it in the balance center. The ac electrodes have inner and outer diameters of 8 and $14 \mathrm{~mm}$, respectively, and an inclination of $18^{\circ}$. The broad shape was chosen in order to reduce field distortions, especially due to the ring supports, while keeping the stabilization strength constant of the EDB high. The conical 
shape of both surfaces of each ac electrode minimizes the range of scattering angles obscured by the ac electrodes. Near the trap center the potential can be approximated by

$V(R, Z)=V_{\mathrm{ac}}\left(c_{-1}+c_{1}\left(Z^{2}-\frac{R^{2}}{2}\right)\right) \cos \omega t$

where $V_{\mathrm{ac}}$ is the amplitude of the ac voltage applied to the electrodes, $\omega$ is the angular frequency of the ac voltage, and $c_{-1}$ and $c_{1}$ are constants. The stabilization strength constant $c_{1}=-1.625 \cdot 10^{4} \mathrm{~m}^{-2}$ was determined by numerically calculating the potential along the trap axis for the given geometry and fitting to it a polynomial of second order for distances up to $1 \mathrm{~mm}$ from the trap center. The parameters $c_{0}$ and $c_{1}$ correspond to the values $C_{0}=0.77$ and $C_{1}=-2.15$ of the dimensionless constants for levitation strength and stabilization strength introduced by Hartung and Avedisian (1992). Particles for injection are picked up from a metal surface using a tungsten needle with $125 \mu \mathrm{m}$ diameter and $5 \mu \mathrm{m}$ radius of curvature at the tip, mounted on a 3-axis translation stage. Particle collection can be made easier by applying to the needle a small positive voltage of 2 to $5 \mathrm{~V}$. For ejection the needle with the particle is positioned just above the aperture. The particle is contact charged by applying to the needle a voltage positive with respect to the aperture. This voltage is increased until the electrostatic force on the particle exceeds the adhesion force (which is large in comparison with gravity) and the particle is released. Using this method, single $5 \mu \mathrm{m}$ silica spheres are ejected at $\sim 100 \mathrm{~V}$ and they obtain charges of typically $\sim 10 \mathrm{fC}$. For particle injection the voltages on the ring electrodes (frequency and amplitude) and the dc electrodes (sign and amplitude) have to be adjusted carefully taking into consideration the size and material of the particle to be injected (see sections $3 \mathrm{~A}$ and $4 \mathrm{C}$ ). Once the particle is trapped in the EDB, it can be moved to the center by adjusting the dc voltage. By applying suitable voltages it is possible to recover the particle back onto the needle for further investigation by other methods, such as SEM.

\section{PARTICLE INJECTION AND RECOVERY A. Particle injection}

When contact charged, particles leaving the needle are exposed to the combined ac and dc fields, the aerodynamic drag force and gravity:

$m \frac{\mathrm{d}^{2} \mathbf{r}}{\mathrm{d} t^{2}}=q \mathbf{E}(\mathbf{r}, t)+m g \mathbf{e}_{z}+\mathbf{F}_{\mathrm{D}}$

In this equation $m, q$ and $\mathbf{r}$ are the particle mass, charge and position, respectively, $\mathbf{E}$ is the electric field, $g$ the acceleration due to gravity, $\mathbf{e}_{z}$ the unit vector parallel to the force of gravity and $\mathbf{F}_{\mathbf{D}}$ the drag, which is parallel but opposite in direction to the velocity of the particle with respect to the medium, defined by

$$
\mathrm{F}_{\mathrm{D}}=-\frac{1}{2} D A \rho_{\mathrm{m}}\left(\frac{\mathrm{d} \mathbf{r}}{\mathrm{d} t}\right)\left|\frac{\mathrm{d} \mathbf{r}}{\mathrm{d} t}\right|
$$

where $D$ is the drag coefficient, $A$ is the projected area of the particle on the plane normal to the direction to its movement, $\rho_{\mathrm{m}}$ is the density of the medium, and $\mathrm{d} \mathbf{r} / \mathrm{d} t$ is the velocity of the particle (the air in the EDB is assumed to be stationary).

For low Reynolds numbers $R e$, the drag coefficient can be approximated by Stokes law, and the drag coefficient is

$D=\frac{24}{R e}$

with

$$
R e=\left|\frac{\mathrm{d} \mathbf{r}}{\mathrm{d} t}\right| \frac{l_{\mathrm{c}} \rho_{\mathrm{m}}}{\mu}
$$

where $l_{\mathrm{c}}$ is the characteristic length of the particle (e. g. the diameter of a sphere or a cylinder), and $\mu$ is the viscosity of the surrounding medium.

Graph a) in Fig. 1 shows the numerically calculated field distribution $E_{\mathrm{ac}}(\mathrm{Z})$ at $t=\mathrm{n} 2 \pi / \omega, \mathrm{n}$ integer, for a voltage $V_{\mathrm{ac}}(t)=1000 \mathrm{~V} \cos (\omega t)$ applied to the ring electrodes. This graph has a nearly linear range between -0.4 and $0.4 \mathrm{~cm}$ which is antisymmetric with respect to $Z=0$. In this range the sinusoidally time varying electric force is approximately proportional to the distance from the center, and an injected particle would oscillate with a damped motion towards the center, if gravity was 
compensated and starting velocity small. However, due to the ac field a particle entering the chamber through the aperture in the top electrode at $Z=-1.15 \mathrm{~cm}$ experiences a force directed towards the top electrode in the range down to $Z=-0.5 \mathrm{~cm}$. If the mass and starting velocity of the particle from the tip are not high enough, the particle can be prevented from entering the trap region around the center of the EDB. This problem can be overcome by increasing the ac frequency or decreasing the ac amplitude. In the case of very light particles it may be necessary to apply an accelerating voltage between the dc electrodes. Curves a) to d) in Fig. 2 show calculated trajectories for $5 \mu \mathrm{m}$ silica spheres with a charge of $10 \mathrm{fC}$ for different potentials of the dc electrodes. The ac voltage at the ring electrodes was set to $1000 \mathrm{~V}$ amplitude. An acceleration voltage of $\sim 30 \mathrm{~V}$ is necessary to transfer the particle into the central trap region (trajectory c). At higher acceleration voltages $(\sim 40 \mathrm{~V})$ the ac field is too weak to trap the particle, which will leave the trap at the opposite side (trajectory d). The particle can be trapped by switching the acceleration field off after the particle entered the trapping region.

\section{B. Particle recovery}

Particles can be recovered from the trap by the following procedure. A voltage is applied to the dc electrodes which is about twice as high as the voltage which is necessary to keep the particle in the trap center, so that the particle oscillates below the upper ring electrode. The needle is then slowly inserted into the trap until the distance between needle tip and trap center is reduced to $6.5 \mathrm{~mm}$, while keeping the needle near ground potential. Before releasing the particle from the trap by switching the ac voltage off, the needle tip has to be aligned exactly on the axis of the balance (this can be checked by a comparison with the position of the particle), and a suitable dc voltage has to be applied to the needle. The ratio between potentials at the needle and at the dc electrodes is chosen in such a way that the field at larger distances from the tip (> $2.5 \mathrm{~mm}$ ) is identical with the field in absence of the needle (Fig. 1, graphs b,c). Needle potentials of lower absolute value would cause repulsion of the particle from axis. In order to prevent particle bouncing or damage during impact the final velocity of the particle should not be too high. On the other hand, the particle should move to the needle in a reasonable, not too long time, and 1 to 2 seconds was found to be satisfactory. Fig. 2e) shows the particle position $Z(t)$ for a 5 $\mu \mathrm{m}$ silica sphere with a charge of $6 \mathrm{fC}$. If necessary, the final acceleration of the particle could be further reduced by using a needle for particle launching different from the one for particle recovery, the latter needle having a larger radius of tip curvature.

\section{STABILITY CHARACTERISTICS OF CYLINDRICAL FIBERS IN THE EDB}

Levitation of nonspherical particles is of interest for several applications, e.g. light scattering measurements, and particle orientation and stability are important in this context. In this work, the stability of levitated cylindrical fibers is investigated. Apart from translational (vibrational) instabilities, angular (rotational) instabilities are observed. Usually, angular instabilities appear first when changing ac parameters of a stable levitated cylindrical particle. In the following, the equations of angular and translational motion are derived and solved numerically, and the results are compared with experiments.

\section{A. Angular stability boundary}

The torque exerted on a fiber positioned in the balance center (which means that the dc field compensates gravity) is:

$\mathbf{M}=\mathbf{M}_{\mathrm{El}}+\mathbf{M}_{\mathrm{D}}$

where $\mathbf{M}_{\mathrm{El}}$ is a torque due to electrostatic forces, and $\mathbf{M}_{\mathrm{D}}$ is a reduction of torque due to aerodynamic drag forces.

For calculating the torque due to the electrostatic forces, the fiber charge $q$ is assumed to be uniformly distributed along the fiber length $L_{\mathrm{p}}$. While this is a simplifying assumption, numerical calculations show that the oscillations of the ring voltage do not significantly alter the charge distribution along the fibers and the charge density near the ends of the fiber is only slightly higher than in the center. This is because for sufficiently large charges on the fibres, which was the case for the experiments reported here, the distribution is determined mainly by Coulomb repulsion on the fiber surface. Also, the polarization charge induced by the ac field is expected to be about three orders of magnitude lower than the charge introduced to the fiber by contact charging. A length element $\mathrm{d} l$ of the fiber placed in the electric potential $V$ and at a distance $l$ from the pivot at $L_{\mathrm{p}} / 2$ experiences a torque 
$\mathrm{d} \mathbf{M}_{\mathbf{E}}=-l(\mathbf{e} \times \nabla V) \frac{q}{L_{p}} \mathrm{~d} l$

where $\mathbf{e}=\sin \theta \mathbf{e}_{R}+\cos \theta \mathbf{e}_{Z}$ is a unit vector parallel to the fiber, with $\mathbf{e}_{R}$ and $\mathbf{e}_{Z}$ being the unit vectors of the radial and axial cylindrical coordinates, respectively, and $\theta$ is the angle between the fibre and the axis of the trap ( $Z$ axis).

The dc field is neglected as it is comparatively weak and, furthermore, it is symmetric near the EDB center and does not therefore exert a torque on the fiber (in the absence of polarization). After the substitution of the ac potential (1), the magnitude of the torque element described by Eq. (7) becomes

$\mathrm{d} M_{E l}=\frac{3 c_{1} q V_{a c} \sin \theta \cos \theta \cos \omega t}{L_{p}} l^{2} \mathrm{~d} l$

A particle subjected to a constant torque $M_{\mathbf{c}}$ due to external forces as well as to viscous drag will attain an equilibrium angular velocity

$$
\frac{\mathrm{d} \theta}{\mathrm{d} t}=B_{\theta} M_{c}
$$

where $B_{\theta}$ is the angular mobility (Fuchs, 1964; Lilienfeld, 1985). Eq. (9) can be considered to be an angular motion equivalent of the Stokes law. The angular mobility for a fibrous particle can be approximated by that of a prolate ellipsoid (Fuchs, 1964)

$$
B_{\theta} \approx \frac{3\left[\frac{2 \beta^{2}-1}{\sqrt{\beta^{2}-1}} \ln \left(\beta+\sqrt{\beta^{2}-1}\right)-\beta\right]}{2 \pi \mu\left(2 R_{\mathrm{e}}\right)^{3}\left(\beta^{4}-1\right)}=\frac{3 C_{\theta}(\beta)}{2 \pi \mu\left(2 R_{\mathrm{e}}\right)^{3}}
$$

where $\beta$ is the ellipsoid aspect ratio, assumed to be equal to that of the cylinder (i.e. $\beta=L_{\mathrm{p}} / 2 R_{\mathrm{p}}$ ) and $C_{\theta}(\beta)$ is a function of $\beta$ only. The ellipsoid and the cylinder are also chosen to have equal volumes, hence the minor semi-axis of the ellipsoid $R_{\mathrm{e}}=(3 / 2)^{1 / 3} R_{\mathrm{p}}$.

$$
\begin{aligned}
& \quad \text { Since at equilibrium } M_{\mathrm{c}}+M_{\mathrm{D}}=0 \text {, we can rewrite Eq. (9) as } \\
& M_{D}=-\frac{1}{B_{\theta}} \frac{\mathrm{d} \theta}{\mathrm{d} t}
\end{aligned}
$$

We use this term as an approximation for the torque due to viscous drag. Newton's second law for angular motion, using the expression for the torque given by Eq. (6), then yields the following differential equation:

$$
2 \frac{\mathrm{d}^{2} \theta}{\mathrm{d} t^{2}} \int_{0}^{\frac{L_{\mathrm{p}}}{2}} l^{2} \rho_{\mathrm{p}} \pi R_{\mathrm{p}}^{2} \mathrm{~d} l=6 V_{\mathrm{ac}} \cos \omega t \frac{q}{L_{\mathrm{p}}} c_{1} \sin \theta \cos \theta \int_{0}^{\frac{L_{\mathrm{p}}}{2}} l^{2} d l-\frac{1}{B_{\theta}} \frac{\mathrm{d} \theta}{\mathrm{d} t}
$$

where $\rho_{\mathrm{p}}$ is the fiber density. The left-hand side of this equation is the moment of inertia of a slender fiber (approximated for $\beta>>1$ ). The first term on the right-hand side is the torque $M_{\mathrm{El}}$ due to the electrostatic forces, obtained by integrating Eq. (8) along the length of the fiber. The second term, the torque reduction due to the aerodynamic drag forces on the entire fiber, is obtained from Eq. (11). After introducing a generalized time variable $T=\omega t$ the following equation of motion for the orientation angle of the fiber is obtained:

$$
\frac{\mathrm{d}^{2} \theta}{\mathrm{d} T^{2}}-\frac{3 c_{1} q V_{a c}}{2 m \omega^{2}} \sin 2 \theta \cos T+\frac{12}{m B_{\theta} L_{p}^{2} \omega} \frac{\mathrm{d} \theta}{\mathrm{d} T}=0
$$

By introducing a stabilizing field strength parameter for angular stability $\varepsilon_{\theta}$

$\varepsilon_{\theta}=-\frac{3 c_{1} q V_{a c}}{2 m \omega^{2}}$

and a drag parameter $\delta_{\theta}$

$\delta_{\theta}=\frac{12}{m B_{\theta} L_{p}{ }^{2} \omega}$

which include all characteristics of the particle and the balance, a generalized equation is obtained: 


$$
\frac{d^{2} \theta}{d T^{2}}+\varepsilon_{\theta} \sin 2 \theta \cos T+\delta_{\theta} \frac{d \theta}{d T}=0
$$

This equation, which is similar to Mathieu's equation, describes the angular motion of any cylindrical fiber positioned in the center of an EDB. It is analogous to the equations derived by Hartung and Avedisian (1992) for the vertical and radial movement of a particle in an EDB.

Eq. (14) allows numerical calculation of stability boundaries for a cylindrical particle in an EDB. A generalized stability diagram is shown in Fig. 3. Due to damping, angular movement of a cylindrical particle should be absent for all values $\left[\delta_{\theta} \varepsilon_{\theta}\right]$ within a stable region. When entering an "instability region", the particle starts to oscillate around its stable orientation at an angular frequency $\omega / 2$. The oscillation is restricted to one plane, which occasionally rotates slowly around the trap axis. The oscillation amplitude will increase when moving deeper into the instability region, until the orientation switches irregularly by multiples of $\pi / 2$. When moving further into the instability region, switching becomes more frequent, and at certain values of ac amplitude and frequency axial instabilities occur (see next section).

According to Eq. (13) a reduction of the drag parameter will cause the angular stability limit to be reached at lower ac amplitudes and higher ac frequencies. For particles of equal mass to charge ratio

$\delta_{\theta} \propto 1 /\left(L_{\mathrm{p}}^{2} \beta C_{\theta}(\beta)\right)$

is obtained. With increasing aspect ratio the factor $1 / \beta C_{\theta}(\beta)$ will increase. On the other hand, $\delta_{\theta}$ will decrease with increasing particle length. For particles which have equal mass and charge the proportionality

$\delta_{\theta} \propto \frac{R_{\mathrm{p}}}{\beta^{2} C_{\theta}(\beta)}$

is obtained. $R_{\mathrm{p}}$ can be expressed by the particle volume $V$ and the aspect ratio:

$$
R_{\mathrm{p}}=\sqrt[3]{\frac{V}{2 \pi \beta}}
$$

Inserting Eq. (17) into Eq. (16) gives

$$
\delta_{\theta} \propto \frac{1}{\beta^{7 / 3} C_{\theta}(\beta)}
$$

Since this expression varies slowly with $\beta$ the drag term depends only weakly on the aspect ratio.

\section{B. Axial and radial stability boundaries}

Apart from angular instabilities, the cylindrical fibers show also instabilities due to translational oscillations parallel to the axis of the EDB, similar to those observed for spherical particles (see e.g. Hartung and Avedisian, 1992). Radial instabilities do not occur due to the smaller gradient of the radial field component: to induce radial instability, higher voltages or lower frequencies are needed than for the axial direction. Once the particle reaches the axial stability boundary, it will oscillate with $\omega / 2$ in the axial direction. When moving further into the instability region, the amplitude of the oscillations will increase until the particle escapes. We have found that an elongated particle originally aligned parallel to the trap axis might become axially unstable at certain values of ac voltage and frequency and flick into the horizontal orientation, where it will be stable due to the larger projected area perpendicular to the trap axis.

The motion of the particle is assumed to be confined in one plane parallel to the axis of the EDB, which is chosen to be the Z-axis. In cylindrical coordinates, the axial component of Eq. (2) is

$m \frac{\mathrm{d}^{2} Z}{\mathrm{~d} t^{2}}=q E_{Z}(R, Z, t)+m g-F_{\mathrm{D} z}$

where $F_{\mathrm{D} z}$ is the axial component of the viscous drag. The drag experienced by an ellipsoid is $F_{\mathrm{D}}=6 \pi \mu R_{\mathrm{s}} u$

where $u$ is the velocity of the ellipsoid and $R_{\mathrm{S}}$ is the radius of a sphere which would experience the same drag. For estimating drag the fiber is assumed to be aligned perpendicularly to the trap axis and 
angular oscillations are neglected. The radius of the "equivalent sphere" for a prolate ellipsoid which is moving perpendicularly to its major axis is

$$
R_{\mathrm{s}}=\frac{8 R_{\mathrm{e}}}{3} \frac{1}{\left[\frac{\beta}{\beta^{2}-1}+\frac{2 \beta^{2}-3}{\left(\beta^{2}-1\right)^{3 / 2}} \ln \left(\beta+\sqrt{\beta^{2}-1}\right)\right]}
$$

Where $\beta$ is the ellipsoid aspect ratio (Happel and Brenner, 1983). When approximating the cylinders by ellipsoids, the same assumptions concerning ellipsoid aspect ratio and volume are made as in section 4A.

By rearranging Eq. (19), inserting Eqs. (7) and (20) and introducing again the generalized time $T$ we obtain the following equation for a fiber for which gravity is compensated by the de field

$$
\frac{\mathrm{d}^{2} Z}{\mathrm{~d} T^{2}}-\varepsilon_{Z} Z(T) \cos (T)+\delta_{Z} \frac{\mathrm{d} Z}{\mathrm{~d} T}=0
$$

The drag parameter $\delta_{z}$ and the stabilizing field strength parameter $\varepsilon_{z}$ for axial stability are, respectively:

$$
\delta_{\mathrm{Z}}=\frac{16 \pi \mu \sqrt[3]{\frac{3}{2}} R_{\mathrm{p}}}{\omega m} \frac{1}{\left[\frac{\beta}{\beta^{2}-1}+\frac{2 \beta^{2}-3}{\left(\beta^{2}-1\right)^{3 / 2}} \ln \left(\beta+\sqrt{\beta^{2}-1}\right)\right]}=\frac{16 \pi \mu \sqrt[3]{\frac{3}{2}} R_{\mathrm{p}} C_{\mathrm{z}}(\beta)}{\omega m}
$$

$\varepsilon_{Z}=-\frac{2 c_{1} q V_{\mathrm{ac}}}{m \omega^{2}}$

where $C_{z}(\beta)$ is a function of the aspect ratio only. Fig. 4 shows the axial stability boundary calculated from Eq. (21). A reduction of the drag term in Eq. (21) will cause the axial stability limit to be reached at lower ac amplitudes and higher frequencies. Considering particles of equal mass to charge ratio

$\delta_{z} \propto \frac{C_{\mathrm{z}}(\beta)}{R_{\mathrm{p}}{ }^{2} \beta}$

is obtained. With increasing aspect ratio the factor $C_{z}(\beta) / \beta$ will decrease. The drag parameter $\delta_{z}$ will decrease with increasing cylinder radius. For particles which have equal mass as well as charge

$\delta_{\mathrm{z}} \propto R_{\mathrm{p}} C_{\mathrm{z}}(\beta) \propto \beta^{-1 / 3} C_{\mathrm{z}}(\beta)$

is obtained. In this expression the drag term increases with aspect ratio.

\section{Experimental investigation of angular and axial stability boundaries}

Angular and axial stability boundaries were investigated experimentally for levitated cylindrical glass fibers. The fibers were selected from a sample of crushed glass fiber loft insulation using a fine tungsten needle mounted on a 3-axis translation stage and deposited initially on a 1000 mesh copper TEM grid. Single fibers were injected into the trap using a method similar to that described in sections 2 and 3A. A fiber was picked up from the grid using a tungsten needle so that it was attached with one end to the needle tip and aligned parallel to the trap axis when it was positioned just above the aperture. Prior to injection its dimensions were measured by optical microscopy. An ac voltage of about $900 \mathrm{~V}$ rms and $400 \mathrm{~Hz}$ was applied to the ac electrodes. An accelerating voltage of about $20 \mathrm{~V}$ was applied to the dc electrodes. In order to obtain high particle charges, the dc voltage at the needle was slowly increased until the particle was ejected. For the fibers used in the experiments described below, the maximum needle voltage varied between 140 and $520 \mathrm{~V}$. When the particle passed the trap centre, the acceleration voltage at the dc electrodes was switched off, and the ac voltage and frequency were increased and decreased, repectively, in order to increase particle stabilization by the trap. The stability limits were determined by a method analogous to the Sprungpunkt technique described for spherical particles (e.g. Hartung and Avedisian, 1992), as follows. The fibers were stably levitated and gravity was compensated by the dc voltage, which means that the fiber was aligned at the trap center either perpendicular or parallel to the trap axis and it was not oscillating axially or rotationally. In the next step the ac amplitude was increased to the maximum available value while keeping the ac frequency reasonably high in order to sustain particle stability. Then the ac frequency was decreased until angular oscillations started, and the onset values were determined. Subsequently, the ac frequency was further 
decreased. The angular oscillations became stronger, and at a certain ac frequency value axial instabilities started. To find further points on the stability boundaries, the ac amplitude was decreased and new frequency values determined. Fig. 5 shows an image of a $136 \mu \mathrm{m}$ long fiber undergoing angular oscillations with an amplitude of about $38^{\circ}$.

Experimental values for the angular stability boundary for cylindrical glass fibers of 40, 83, 89 and $144 \mu \mathrm{m}$ length are given in Fig. 3. The particle diameter varied between 8.1 and $11.2 \mu \mathrm{m}$. The frequency and voltage values $\left[f, V_{\text {ac }}\right]$ for the angular stability limit for the different fibers as well as individual fiber stability boundaries corresponding to the calculated graph in Fig. 3 are shown in Fig. 6. The parameters of the investigated particles are listed in Table 1 . The particle mass was determined from the particle dimensions using the density value of $2.2 \mathrm{~g} / \mathrm{cm}^{3}$, and the particle charge was calculated from the dc voltage $V_{\mathrm{dc}}$ necessary to compensate for gravity using the equation $q=m g /\left(c_{0} \cdot V_{\mathrm{dc}}\right)$.

Fig. 3 shows that, apart from slightly higher $\varepsilon_{\theta}$ values for the $40 \mu \mathrm{m}$ fiber, the data points for the fibers of different length are aligned along the same curve, which has the same slope as the calculated graph. Most data points lie within experimental error of the calculated graph, which is also true for the $40 \mu \mathrm{m}$ fiber. For this fiber the experimental error of the field strength parameter is largest due to the high particle charge, which results in the smallest value of $V_{\mathrm{dc}}$ and therefore the highest relative error of measurement. For the 83 and $89 \mu \mathrm{m}$ fibers, which have approximately equal mass but a charge ratio of $1.83: 1$, the angular stability limit was found to be at adjacent frequency ranges (300 to $170 \mathrm{~Hz}$ and 170 to $120 \mathrm{~Hz}$, respectively) for the investigated ac voltage ranges (1700 to $700 \mathrm{~V}$ rms and 1530 to $1080 \mathrm{~V}$ rms, respectively). The 83 and $144 \mu \mathrm{m}$ fibers, which have approximately equal mass and charge but aspect ratios of 7.38 and 17.14 , respectively, show nearly identical values $\left[f, V_{\text {ac }}\right]$ for the angular stability limit. This agrees with Eq. (18), which predicts only a small increase of the drag term with aspect ratio.

In Fig. 4 the data points for the axial stability limit of cylinders of different length are aligned along a curve which is parallel to the calculated graph, although the measured field strength parameter values and/or drag parameter values are slightly higher than the calculated ones. The values $\left[f, V_{\mathrm{ac}}\right]$ for the axial stability limit for the different fibers as well as frequency-voltage curves obtained using the values $\left[\delta_{z}, \varepsilon_{z}\right]$ from the calculated graph in Fig. 4 are shown in Fig. 6. For each fiber a linear dependence between ac frequency and amplitude is observed in the investigated parameter range. Like for the angular stability limit, the $\left[f, V_{\text {ac }}\right]$ values for the 83 and $144 \mu \mathrm{m}$ fibers are aligned nearly along the same line. This is consistent with Eq. (23) which predicts only a slight change in the drag with the aspect ratio. For the 83,89 and $144 \mu \mathrm{m}$ fibers the slope of the experimental data is slightly steeper for the axial stability limit than for the angular stability limit. This means that at the onset of axial instabilities angular oscillations are becoming stronger with increasing ac amplitude. It can be expected that for certain low values of ac frequency and amplitude the angular and axial stability limits will occur simultaneously and that below these points axial instabilities will appear first. In principle, this agrees with the calculated curves in Fig. 6, although the intersections of the calculated stability boundaries for the different fibers and the intersection locations extrapolated from experimental data are different due to experimental errors.

\section{CONCLUSIONS}

An EDB for single particle injection, levitation and recovery has been developed. The particle is contact charged on the tip of a sharp tungsten needle. Especially for the injection of small (and therefore light) particles, amplitude and frequency of the ac voltage applied to the ring electrodes and the dc voltage applied to the end-cap electrodes have to be chosen carefully in order to allow the particle to reach the trapping region of the EDB. After levitation experiments the particle can be recovered from the trap by applying suitable dc potentials to the needle tip and the end caps and switching the ac voltage off.

Stability characteristics of cylindrical fibers in this EDB have been investigated, including angular as well as translational motion. Because axial and angular instabilities are not independent, it is difficult to predict the onset of angular and/or axial instabilities for a particle of given mass, charge and shape. One possible application of angular oscillations in an electrodynamic trap might be for the characterization of particles by light scattering, for example shape determination. Furthermore, particle instabilities can be used for switching between vertical and horizontal particle orientation, either by inducing strong angular oscillations and moving back into a stable region when the particle is oscillating around the required orientation, or by passing through axial instabilities of relatively large amplitude. For a completely rotationally symmetric trap the orientation of the particle in the plane 
parallel to the ring electrodes would not be defined. However, in the trap used in the experiments described here, the particle will align perpendicularly to the supports for the ring electrodes. In this way observation and measurement at two orthogonal particle orientations is possible. The particle can be aligned in a direction approximately perpendicular to these two orientations if the rings are rotated by $90^{\circ}$. Then the particle can be switched between an alignment parallel to the axis of the rings, which is now horizontal, and a direction in the plane of the rings. In a perfectly rotationally symmetric trap a particle of perfect cylindrical symmetry would align horizontally. However, irregularities and/or inhomogeneities may cause the particle to be slightly tilted. Intermediate angles of inclination of the particle can be achieved by corresponding tilting of the ring electrodes.

\section{ACKNOWLEDGEMENTS}

This research was supported by the grant GST/02/2321 from the Natural Environment Research Council. We are indebted to John Kerr and Richard White for technical assistance.

\section{REFERENCES}

Aardahl, C.L., Vehring, R., Davis, E.J., Schweiger, G. and Swanson, B.D. (1997) Trapping twoparticle arrays in a double-ring electrodynamic balance. J. Aerosol. Sci. 28(8), 1491.

Arnold, S. and Folan, L.M. (1986) Fluorescence spectrometer for a single electrodynamically levitated microparticle. Rev. Sci. Instrum. 57(9), 2250.

Arnold, S. and Hessel, N. (1985) Photoemission from single electrodynamically levitated microparticles. Rev. Sci. Instrum. 56(11), 2066.

Ataman, S. and Hanson, D.N. (1969) Measurement of charged droplets. Ind. Eng. Chem. Fundam. 8, 833.

Davis, E.J. (1997) A history of single aerosol particle levitation. Aerosol Sci. Technol. $26,212$.

Frickel, R.H., Shaffer, R.E., Stamatoff, J.B. (1978) Containment of charged aerosol particles. Report $A D / A 056$ 236, US Department of Commerce, Springfield. Virginia: National Technical Information Service.

Fuchs, N.A. (1964) The mechanics of aerosols. Pergamon Press, Oxford.

Happel, J. and Brenner, H. (1983) Low Reynolds number hydrodynamics with special applications to particulate media. Martinus Nijhoff Publishers, The Hague.

Hartung, W.H. and Avedisian, C.T. (1992) On the electrodynamic balance. Proc. R. Soc. Lond. A 437, 237.

Laucks, M.L., Roll, G., Schweiger, G. and Davis, E.J. (2000) Physical and chemical (Raman) characterization of bioaerosols -pollen. J. Aerosol Sci. 31(3), 307.

Lilienfeld, P.J. (1985) Rotational Electrodynamics of Airborne Fibres. J. Aerosol Sci. 16 (4), 315.

Sasse, C., Muinonen, K., Piironen, J. and Droese, G.J. (1996) Albedo measurements on single particles. Quant. Spectrosc. Radiat. Transfer 55(5), 673.

Straubel, H. (1956) Die Dosierung von Substanzmengen unter $10^{-6} \mathrm{~g}$ mittels elektrostatischer Aufladung für Zwecke der Mikroanalyse. Z. Elektrochem. 60 (9/10), 1033.

Vedder, J.F. (1963) Charging and Acceleration of Microparticles. Rev. Sci. Instrum. 34 (11), 1175.

Wuerker, R.F., Shelton, H. Langmuir, R.V. (1959) Electrodynamic containment of charged particles. J. Appl. Phys. 30 (3), 342.

Zheng, F., Laucks, M.L. and Davis, E.J. (2000) Aerodynamic particle size measurement by electrodynamic oscillation techniques. J. Aerosol Sci. 31(10), 1173. 
Table 1. Parameters of levitated glass fibers

\begin{tabular}{ccccccc}
\hline Particle No & $\begin{array}{c}\text { Length } L_{\mathrm{p}} \\
(\mu \mathrm{m})\end{array}$ & $\begin{array}{c}\text { Diameter } 2 R_{\mathrm{p}} \\
(\mu \mathrm{m})\end{array}$ & $L_{\mathrm{p}} /\left(2 R_{\mathrm{p}}\right)$ & Mass $m$ & Charge $q$ & $q / m$ \\
& 40 & 8.1 & $(\mathrm{ng})$ & $(\mathrm{pC})$ & $(\mathrm{pC} / \mathrm{ng})$ \\
\hline 1 & 83 & 11.2 & 7.41 & $18.2 \pm 0.54$ & $0.347 \pm 0.039$ & 0.0191 \\
3 & 89.2 & 11.2 & 7.96 & $19.5 \pm 0.54$ & $0.152 \pm 0.009$ & 0.0078 \\
4 & 144 & 8.4 & 17.14 & $17.6 \pm 1.26$ & $0.356 \pm 0.028$ & 0.0202 \\
\hline
\end{tabular}

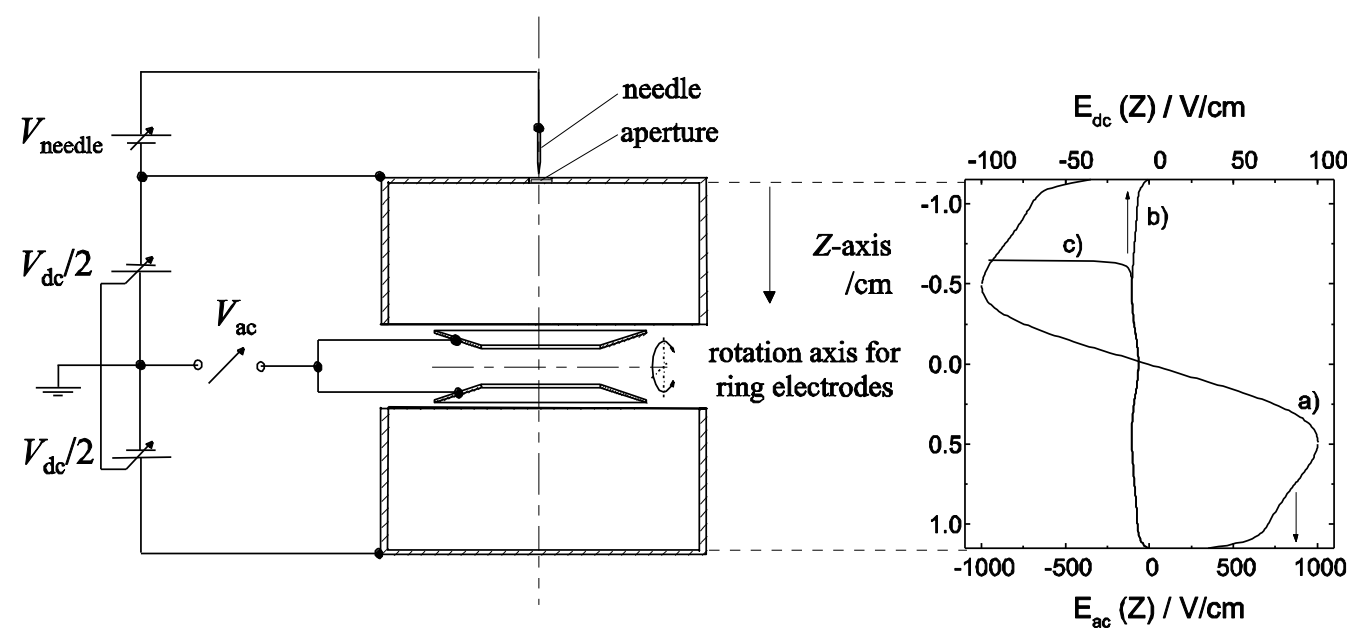

Fig. 1. Diagram of the EDB and electric field strength distributions along the trap axis: a) ac field amplitude $E_{\mathrm{ac}}(Z)$ for an ac voltage of $1000 \mathrm{~V}$ amplitude applied to the ring electrodes, b) field strength of the dc field $E_{\mathrm{dc}}(Z)$ along the trap axis for $-10 \mathrm{~V}$ and $+10 \mathrm{~V}$ w. r. t. ground potential at the top and bottom dc electrode, respectively, c) $E_{\mathrm{dc}}(Z)$ along trap axis for needle position at $0.65 \mathrm{~cm}$ above the trap center for particle recovery, $V_{\text {needle }}=-7 \mathrm{~V}$, with dc electrodes at the same potential as in $\mathrm{b}$ ). 


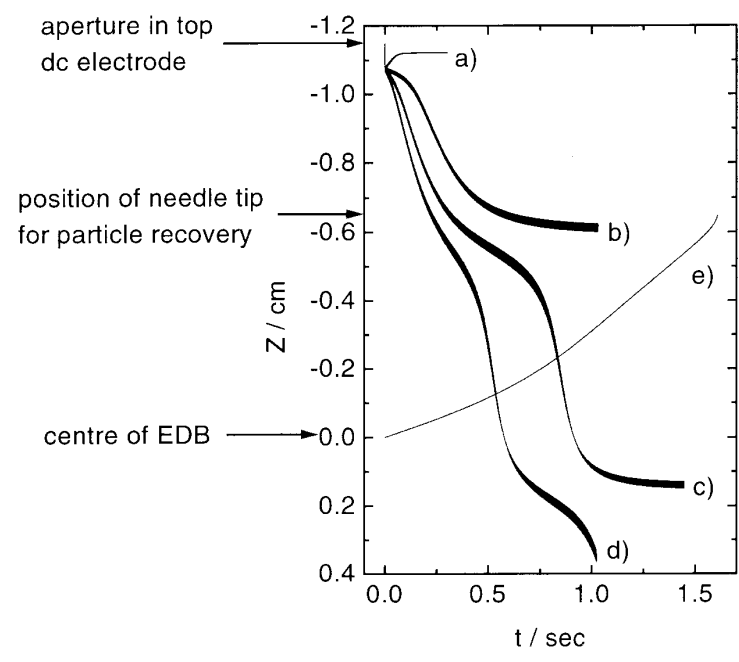

Fig. 2. Calculated launching trajectory of a $5 \mu \mathrm{m}$ silica sphere $(q=10 \mathrm{fC})$ for a ring electrode voltage $V_{\text {ac }}=1000 \mathrm{~V}$ amplitude, $f=800 \mathrm{~Hz}$ and an accelerating voltage $V_{\text {dc }}$ between the dc electrodes of a) $0 \mathrm{~V}$, b) $20 \mathrm{~V}$, c) $30 \mathrm{~V}$, d) $40 \mathrm{~V}$. Graph e) shows the calculated trajectory for recovery of a $5 \mu \mathrm{m}$ sphere $(q=$ $6 \mathrm{fC})$ at the needle tip positioned at $-0.65 \mathrm{~cm}\left(\mathrm{~V}_{\text {needle }}=-7 \mathrm{~V}\right)$, potentials at top and bottom dc electrode were set to $-10 \mathrm{~V}$ and $10 \mathrm{~V}$, respectively.

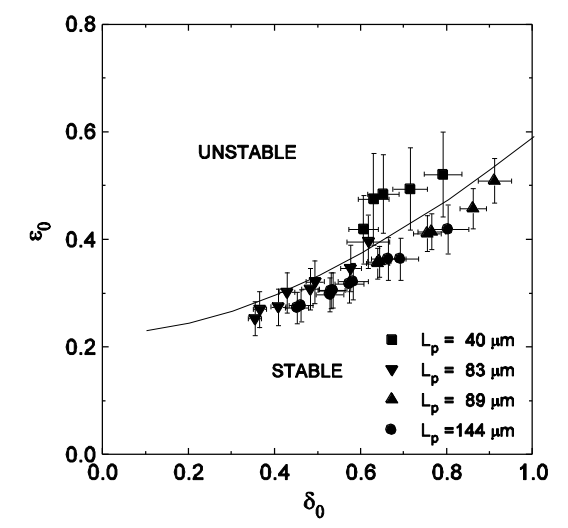

Fig. 3. Calculated angular instability boundary (Eq. (14)), and experimental results for glass fibers 40 , 83, 89, and $144 \mu \mathrm{m}$ in length. 


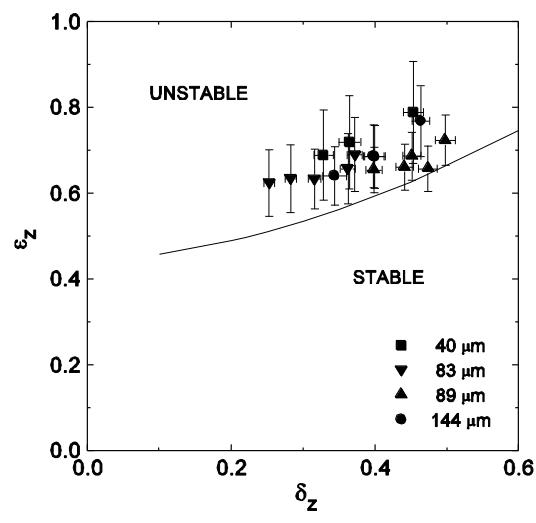

Fig. 4. Calculated axial instability boundary (Eq. (21)), and experimental results for glass fibers 40, 83, 89 , and $144 \mu \mathrm{m}$ in length.

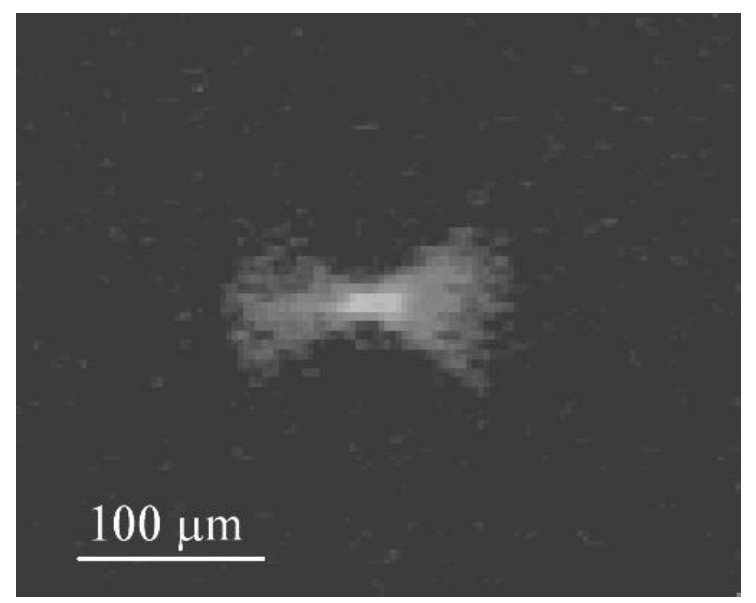

Fig. 5. Angular oscillations of a $136 \mu \mathrm{m}$ long fiber.

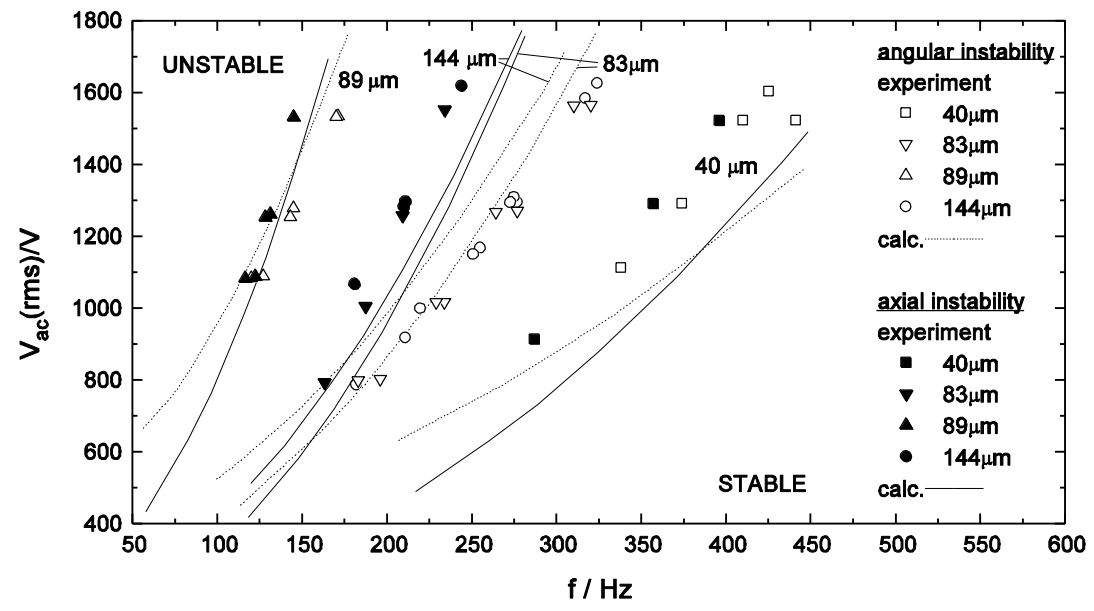

Fig. 6. Experimental values $\left[f, V_{\text {ac }}\right]$ for angular and axial instability boundaries for glass fibers 40,83 , 89 , and $144 \mu \mathrm{m}$ in length and frequency-voltage curves calculated using the values $\left[\delta_{\theta}, \varepsilon_{\theta}\right]$ and $\left[\delta_{z}, \varepsilon_{z}\right]$ of the calculated graphs in Figs. 4 and 5, respectively. 